\title{
A Study of Emotions in Requirements Engineering
}

\author{
Ricardo Colomo-Palacios ${ }^{1, *}$, Adrián Hernández-López ${ }^{1}$, Ángel García-Crespo ${ }^{1}$, \\ and Pedro Soto-Acosta ${ }^{2}$ \\ ${ }^{1}$ Universidad Carlos III de Madrid \\ Av. Universidad 30, Leganés, 28911, Madrid, Spain \\ \{ricardo.colomo, adrian.hernandez, angel.garcia\}@uc3m.es \\ ${ }^{2}$ University of Murcia \\ Campus de Espinardo, 30100, Murcia, Spain \\ psoto@um.es
}

\begin{abstract}
Requirements engineering (RE) is a crucial activity in software development projects. This phase in the software development cycle is knowledge intensive, and thus, human capital intensive. From the human point of view, emotions play an important role in behavior and can even act as behavioral motivators. Thus, if we consider that RE represents a set of knowledge-intensive tasks, which include acceptance and negotiation activities, then the emotional factor represents a key element in these issues. However, the emotional factor in RE has not received the attention it deserves. This paper aims to integrate the stakeholder's emotions into the requirement process, proposing to catalogue them like any other factor in the process such as clarity or stability. Results show that high arousal and low pleasure levels are predictors of high versioning requirements.
\end{abstract}

Keywords: Software Engineering, Requirements, Software psychology, Emotions.

\section{Introduction}

The human dimension is a key element in software engineering (SE), sometimes having greater importance than the technical dimension. Its importance is due to the fact that SE is essentially based on intellectual and social activities [1]. The roles of humans involved in SE fall into four categories; the individual, the team, management and the stakeholder/interested party, including customer and client. There are always interrelations and overlaps among these four categories. According to DeMarco and Lister [2], the "final outcome of any [SE project] effort is more a function of who does the work than of how the work is done" (p. 93), and is defined by the equation effort $=$ people $x$ time . Although the importance of human factors has been widely recognized as key for SE, researchers should place greater focus on the humans involved in SE than what has been done to date [3].

Software requirements express the needs and constraints placed on a software product that contributes to the solution of some real-world problem. The term Requirements Engineering (RE) is widely used in the field to denote the systematic handling of 
requirements. In order to establish a sound requirements definition, we must begin to pay attention to the human dimensions, consciously addressing the psychology and sociology of its use, i.e. improving information requirements determination from a cognitive perspective [4], cognitive bias in SE [5], and cognitive fit in requirements modeling [6]. The importance of human factors in RE is reflected in the fact that regardless of the methods and tools employed, the success of requirements analysis depends on how well users and analysts communicate and collaborate [7]. Furthermore, the participation of the customer in defining the requirements is vital [8]. Finally, it can be said that requirements engineering is a multidisciplinary human-centered process, although we can benefit from some tools and techniques in addition to human expertise [9].

Even if requirements evolution is managed correctly, most software errors are introduced during this phase and emerge at a later stage [10]. According to the review carried out by Walia and Carver[11], many main errors that can occur in other phases are related to the requirements phase. Hence the errors in the requirements are costly and dangerous. Because of the intrinsic importance of requirements, along with strong human influence in the production and development of these requirements, the aim of this paper is twofold. Firstly, the importance of knowing the stakeholders' views on requirements from an emotional standpoint is highlighted. Secondly, through a human emotions specification technique, the method to include this feature in requirements is presented. The ultimate goal is to build an instrument that, through analysis of the emotions present in the requirements, predicts requirements evolution over time and incorporates standards for better governance of the project.

\section{The Importance of Emotions and RE}

Human behavior is influenced not only by rational-economic factors; it is influenced, among other factors, by emotions [12]. Returning to the review conducted by Walia and Carver [11], human errors in the requirements phase were identified and classified into the following categories: communication, participation, domain knowledge, specific application knowledge, process execution, and other cognition errors. In this paper we will focus on the "other cognition" errors, specifically in emotions. Determining a unique definition of the term "emotion" represents a complicated task. In a study at the beginning of the 1980s, Kleinginna \& Kleinginna [13] found 92 definitions and classified them into 11 categories. Leaving aside the discussion concerning the concreteness and universality of emotion concept, Izard [14] claimed that emotion is composed of three aspects: a) the experience or conscious feeling of emotion, b) the processes that occur in the brain and nervous system, and c) the observable extensible patterns of emotion. As a result of this asseveration, Russell, Weiss and Mendelsohn [15] proposed a measure of affect which had a profound impact on social psychology. They termed the measure the Affect Grid, a scale designed as a quick means of assessing affect along pleasure-displeasure and arousal-sleepiness dimensions on a 1-9 scale. According to the studies of these authors, the Affect Grid is potentially suitable for any study that requires judgments about affect of either a descriptive or a subjective kind.

The interaction between requirements and emotions is not totally unexplored. Ramos and Berry [16] have discussed and emphasized the importance of emotions in 
RE. The reasons for their analysis are, firstly, the transformation that involves the use of a new system to users [17], and secondly, the difficulty in defining the requirements in ways that are beneficial for developers and users, i.e. establishing a win-win relationship . The result of their studies confirms the importance and validity of the emotional factor in RE, as well as any other classic factor, such as performance, cost, user interface, etc. The approach proposed in this paper focuses on the evolution of requirements and the parallel evolution of the emotions through the various stages.

From the evolutionary viewpoint within a software development project, requirements evolve from their creation in the elicitation phase. Thus, an overall picture of emotions and requirements should provide the starting point for the creation, development and specification of requirements by taking into account the emotional factor. This paper aims at creating and testing a solution for the prediction of the life cycle of requirements. The ultimate goal is to use emotional qualification of requirements as a key for a more complete requirements management and greater suitability of soft issues in requirements specification.

\section{The Experiment}

\subsection{Research Design}

Based on the idea that our processing of emotions tends to be biased, and that these biases affect our perception, judgment, and behavior [18], this paper proposes using the Affect Grid psychological tool created by Russell et al. [15] to characterize requirements throughout the development process. Thus, based on requirements, stakeholders express the emotion that requirements raise for them. It requirements measurement are intended to be discrete, but their changes over time serialize intake data enabling the creation of patterns.

For each requirement, it is proposed that all stakeholders involved in it perform an emotional assessment about this requirement using the Affect Grid. In addition, it is proposed that this assessment be repeated with each requirement version upgrade. Thus, through the requirements repository, the emotional traceability can be established taking into account the emotional assessment made by stakeholders.

The emotional evaluation is conducted through the Affect Grid, which is a singleitem scale of pleasure and arousal. Thus, the participants answers the question "What's your emotion regarding this requirement definition?" and places one checkmark somewhere in the grid. Subsequently, these data are encoded together with requirements to ensure the pursued emotional traceability.

The proposed evaluation method has been implemented in two projects. The first project, known as Project A, is developed in the context of a software development organization using its own means. The project consists of performing an adaptive maintenance of a legacy information system. To this end, a total of 28 user requirements were identified and documented that subsequently served as the basis for a software development project which lasted 7 months. There were a total of 97 different versions of requirements.

Meanwhile, project B was focused on the development of a touristic information system. In this context, a software development organization was hired to undertake the 
system development. The project team specified a total of 37 user requirements. The development project lasted 6 months, after which the developed system was implemented. There were a total of 115 different requirements versions. All scores were codified using a 1-9 Likert Scale, with 1 meaning low arousal-pleasure, and 9 meaning high arousal-pleasure. Each score represents the pleasure (y axes) and arousal (x axes).

Based on the requirements identified in projects A \& B, both users and developers implement the operations described, namely rating requirements using the Affect Grid in all its versions. The experiment's aim is to verify whether the emotional assessment responds to any pattern. Therefore, some hypotheses are set. In the first place, from the evolution of scores along the versions, two hypotheses are defined:

H1: Higher requirement versions' scores have higher Pleasure scores.

H2: Higher requirement versions' scores have lower Arousal scores.

These hypotheses are based on the premise that requirements finally have to fit users' needs on the one hand, and have to be carried out by developers on the other hand. In addition, initial contacts with requirements produce stress in developers and users. In order to specialize these hypotheses, hypotheses regarding final requirements are set:

H3: Pleasure scores for final requirements are higher than for non-final requirements.

H4: Arousal scores for final requirements are lower than for non-final requirements.

Final requirements are supposed to fit the user's needs and are accepted by all stakeholders; therefore these hypotheses specialize $\mathrm{H} 1$ and $\mathrm{H} 2$.

\subsection{Sample Description}

The sample was composed of 11 individuals: five of them belonging to project $\mathrm{A}$ (3 developers and 2 users), and 6 to project B (3 developers and an equal number of users). In relation to demographic characteristics, the group of participants consisted of 4 women (36\%) and 7 men (64\%), with an average age of 32.7. The projects sample choice was made according to the available projects, and the participants are those directly involved in the requirements process.

\subsection{Results and Discussion}

As a result of participants' activities, a total of 1,175 emotional ratings were collected. Table 1 shows average and standard deviation of Pleasure and Arousal dimensions in different scenarios.

The data analysis of final requirements scores shows that there is a marked trend of Pleasure and Arousal scores in the case of final version requirements. On the one hand, Pleasure dimension remains constant while on the other hand, Arousal decreases with time. Further analysis is needed to shed light on the differences of these results with those obtained for all versions scores, which points to an increase of pleasure along versions and a decrease of arousal. A set of T-test has been carried out between version $\mathrm{x}$ and $\mathrm{x}+\mathrm{n}$. From these tests, it can be concluded that there are not significant changes when $\mathrm{n}$ is low (1 or 2$)$, but it is significant with higher $\mathrm{n}(<=3)$. These results support $\mathrm{H} 1$ and $\mathrm{H} 2$; the Pleasure dimension score increases throughout versions, while the Arousal dimension decreases. But two questions arise from these 
Table 1. Average and Standard Deviation of emotional ratings of requirements

\begin{tabular}{lcccccccc}
\hline Version & \multicolumn{2}{c}{ All } & \multicolumn{2}{c}{ Final } & \multicolumn{2}{c}{ Pleasure (Total) } & \multicolumn{2}{c}{ Arousal (Total) } \\
\hline & $\mathrm{N}$ & Ratings & $\mathrm{N}$ & Ratings & $\mathrm{A}$ & $\mathrm{St}$ & $\mathrm{A}$ & $\mathrm{St}$ \\
\cline { 2 - 9 } 1 & 65 & 362 & 14 & 79 & 4,738 & 1,947 & 5,229 & 1,582 \\
2 & 51 & 283 & 14 & 78 & 4,604 & 1,676 & 4,873 & 1,503 \\
3 & 37 & 205 & 13 & 74 & 4,654 & 1,566 & 4,634 & 1,368 \\
4 & 24 & 131 & 7 & 38 & 5,038 & 1,605 & 4,496 & 1,349 \\
5 & 17 & 93 & 7 & 36 & 5,602 & 1,575 & 4,441 & 1,246 \\
6 & 10 & 57 & 4 & 52 & 5,404 & 1,412 & 4,421 &, 963 \\
7 & 6 & 33 & 4 & 22 & 5,606 & 1,435 & 4,273 &, 876 \\
8 & 2 & 11 & 2 & 11 & 5,455 & 1,128 & 4,364 & 1,12 \\
All & 212 & 1175 & 65 & 362 & 4,856 & 1,737 & 4,821 & 1,462 \\
Final & 65 & 362 & 65 & 362 & 5,718 & 1,517 & 4,602 & 1,299 \\
\hline
\end{tabular}

results and cannot be solved with the data used for this experiment. Firstly, is the temporal distance between versions influencing the emotional rating? And secondly, if it does influence it, how does it influence the significance between versions?

To check whether there are significant differences between non-final requirements' and final requirement's scores, the T-test has been carried out. For this test, the scores of first requirements that are final version requirements at the same time are considered only as final version requirements. The test results determine that there are differences in the Pleasure dimension between final and non final requirements $(\mathrm{t}(1175)=12.537, \mathrm{p}<.05))$, and in Arousal dimension $(\mathrm{t}(1175)=-3.656, \mathrm{p}<.05))$. With these results, $\mathrm{H} 3$ and $\mathrm{H} 4$ are proved. That is, the final requirements, from the emotional point of view, fits best with stakeholder views, generating in them higher Pleasure and lesser Arousal levels than first versions. Thus, requirements evolve from the Stress quadrant to Relaxation and to a lesser extent, to Excitement.

In addition, to verify whether there are significant differences between the scores of users and developers, T-test has been made for each dimension and each version (non-final and final versions). Thus, the comparative level of version one requirements shows that there are no significant differences for Pleasure dimension, but there are significant differences for Arousal $(\mathrm{t}(362)=8.650, \mathrm{p}<.05)$. This may indicate that the stress that developers are facing in the development is higher than users'. On the other hand, users have somewhat higher values in the pleasure dimension, but they are not significant. In relation to the comparisons made in the final versions of requirements, there are no significant differences between user and developer ratings in both, Pleasure and Arousal dimensions. The explanation for this may be that at the end of the process, both developers and users have a comparable level of confidence after reaching agreements and common definitions. Thus, the level of stress decreases greatly and is distributed in a balanced way between both groups.

\section{Conclusions and Future Work}

The study of human emotions and passions is a classic theme in the history of thought. In this paper, following a novel and promising research line, we propose 
integrating emotions into the RE process. As a result, one of the classic tools of analysis and evaluation of emotions is integrated into the categorization of the requirements. This tool has to be included in "informal" techniques for RE [19]. Results show that emotions are a factor to take into account in establishing requirements stability. Thus, knowing the stakeholder's emotions involves understanding the reliability and stability of the definition of those requirements. Moreover, knowing the emotional state of the development team helps the manager to create an environment capable of combating the effects of "bad" emotions. Thus, training the development team on stress management, communication and assertiveness will improves the coping ability of the RE.

As future works, four research lines are proposed. Firstly, it is necessary to apply this study in more projects in order to contrast these results. Secondly, it has to be determined, using the proposed model, if there are differences among different kinds of software development projects in stakeholders' emotional response. Thirdly, it must be determined if organizational climate or work environment within an organization influences the emotional rating of the requirements in the same way that it influences other institutional tasks. Finally, following the tradition of research on emotion in the field of social psychology, a cross cultural study is proposed [20] in order to provide data in relation to this crucial factor in the classic study of emotions and their possible translation into RE.

Two additional questions arise after this study. On the one hand, is the decrease of Arousal equal to the decrease detected for the scores of requirements despite being final, or not? This question responds to the similarity found in the decrease of Arousal in both cases. On the other hand, can the constancy of the Pleasure dimension be considered a key factor for final requirements? Requirements in final versions tend to be scored with 6 in Pleasure dimensions, but there is not enough data to test this question; there are 65 requirements and 1175 scores, 362 scores from final requirements. These two questions will be dealt with in future work.

\section{References}

1. Sommerville, I., Rodden, T.: Human, social, and organizational influences on the software process. In: Fuggetta, A., Wolf, A. (eds.) Trends in software process, pp. 89-110. John Wiley, New York (1996)

2. DeMarco, T., Lister, T.: Peopleware: Productive projects and teams. Dorset House Publishing Co., Inc., New York (1987)

3. Feldt, R., Torkar, R., Angelis, L., Samuelsson, M.: Towards individualized software engineering: Empirical studies should collect psychometrics. Paper presented at the Proceedings of the 2008 international workshop on Cooperative and human aspects of software engineering (CHASE 2008), Leipzig, Germany (2008)

4. Browne, G.J., Ramesh, V.: Improving information requirements determination: A cognitive perspective. Information and Management 39(8), 625-645 (2002)

5. Stacy, W., MacMillan, J.: Cognitive bias in software engineering. Communications of the ACM 38(6), 57-63 (1995)

6. Agarwal, R., Sinha, A.P., Tanniru, M.: Cognitive fit in requirements modeling: A study of object and process methodologies. Journal of Management Information Systems 13(2), 137-162 (1996) 
7. Safayeni, F., Duimering, P.R., Zheng, K., Derbentseva, N., Poile, C., Ran, B.: Requirements engineering in new product development. Communications of the ACM 51(3), 77 $82(2008)$

8. Saiedian, H., Dale, R.: Requirements engineering: Making the connection between the software developer and customer. Information and Software Technology 42(6), 419-428 (2000)

9. Nuseibeh, B., Easterbrook, S.: Requirements engineering: A roadmap. Paper presented at the Proceedings of the Conference on The Future of Software Engineering (ICSE 2000), Limerick, Ireland (2000)

10. Pfleeger, S., Atlee, J.: Software engineering: Theory and practice. Prentice Hall, Upper Saddle River (2006)

11. Walia, G.S., Carver, J.C.: A systematic literature review to identify and classify software requirement errors. Information and Software Technology 51(7), 1087-1109 (2009)

12. Hochschild, A.: The sociology of feeling and emotion: Selected possibilities. Sociological Inquiry 45(2-3), 280-307 (1975)

13. Kleinginna, P.R., Kleinginna, A.M.: A categorized list of emotion definitions, with suggestions for a consensual definition. Motivation and emotion 5(4), 345-379 (1981)

14. Izard, C.E.: Human emotions. Plenum Press, New York (1977)

15. Russell, J.A., Weiss, A., Mendelsohn, G.A.: Affect grid: A single-item scale of pleasure and arousal. Journal of personality and social psychology 57(3), 493-502 (1989)

16. Ramos, I., Berry, D.M.: Is emotion relevant to requirements engineering? Requirements Engineering 10(3), 238-242 (2005)

17. Ramos, I., Berry, D.M., Carvalho, J.Á.: Requirements engineering for organizational transformation. Information and Software Technology 47(7), 479-495 (2005)

18. Kahneman, D., Diener, E., Schwarz, N.: Well-being: The foundations of hedonic psychology. Russell Sage Foundation Publications (2003)

19. Fraser, M.D., Kumar, K., Vaishnavi, V.K.: Informal and formal requirements specification languages: Bridging the gap. IEEE Transactions on Software Engineering 17(5), 454-466 (1991)

20. Mesquita, B., Frijda, N.H.: Cultural variations in emotions: A review. Psychological bulletin 112(2), 179-204 (1992) 